\title{
Astrometry with ground-based interferometers
}

\author{
A. Boden ${ }^{1}$ and A. Quirrenbach ${ }^{2}$ \\ ${ }^{1}$ Michelson Science Center, California Institute of Technolgy \\ Pasadena, CA, USA bode@astro.caltech.edu \\ ${ }^{2}$ Zentrum fur Astronomie, University of Heidelberg \\ Heidelberg, Germany A.Quirrenbach@lsw.uni-heidelberg.de
}

\begin{abstract}
We review the status of ground-based interferometric astrometry. This will include a review of technology and results in differential techniques (e.g. relative orbit determination), as well as global astrometry techniques (globally-registered parallax and proper-motion estimates).
\end{abstract}

Keywords. instrumentation: interferometers, techniques: high angular resolution, techniques: interferometric, astrometry, binaries: general

\section{Introduction}

In this contribution we review the status on long-baseline (LB) optical/near-IR (OIR) interferometric astrometry. Operationally we define a long-baseline interferometer as a device that combines light from multiple independent telescopes to form and measure interference fringes - variations in detected power as a function of optical path difference between two telescopes. This definition excludes our consideration of interferometry using a single telescope aperture (e.g. "speckle" or aperture masking interferometry). And as the title implies we will discuss interferometric astrometry in the optical and near-IR; the many contributions and future potential of interferometric astrometry in radio and $\mathrm{mm}$ bands are discussed in other contributions to these proceedings (e.g. see manuscripts by Reid, Lestrade, and Loinard).

An astronomical interferometer is a device that measures interference (or attributes associated with the interference) in the incident radiation field from an astronomical source. This first such interferometer operating in OIR bands was constructed by Michelson at Mt. Wilson in 1920. The astrometric potential of LB OIR interferometers was appreciated by Michelson (1920) in their development; Anderson (1920) and Merrill (1922) observed a number of binary stars with the Michelson's Mt. Wilson interferometer, and published the first estimates of Capella's visual orbit. Since that time until today LB OIR interferometers have been used extensively in relative astrometry of stars over fields from milliarcseconds to tens of degrees, and when combined with external, global reference frames (e.g. FK5 or Hipparcos) such measurements have been used to compute differential corrections to stellar positions, with hopes of extending these analyses to globally-registered proper motion and parallax estimates.

In what follows we will survey astrometric methods used with LB OIR interferometers, and survey the scientific contributions made with these techniques.

\section{Astrometric methods with interferometers}

LB OIR interferometers perform astrometry of stellar fields by analyzing the fringe patterns of stars measured by the interferometer. (The reader unfamiliar with astronom- 
ical interferometry and interferometric observables is referred to Perley et al. 1989, Shao \& Colavita 1992b, Thompson et al. 2001, Quirrenbach 2001, and Boden 1999a).

Depending on the parameters of the stellar field and the interferometer, the exact analysis methods can be different, but generally all methods fall into two broad classes:

- Fringe amplitude Astrometry: When the fringe patterns from multiple sources overlap (i.e. are within a fringe coherence length), the (power-normalized) fringe amplitude contains information on the relative separation and brightness of the sources. Fringe amplitude analysis necessarily applies when multiple sources are within a coherence length of each other, so this technique is applicable only over very small astrometric fields field sizes of millarcseconds to on the order of 100 millarcseconds (depending on interferometer parameters). With such field sizes these amplitude techniques have typically been used in the resolution and orbital analysis of close binary stars. Notable in fringe amplitude analysis is the necessity to include the relative brightness between the sources in the astrometric analysis.

- Separated fringe packet astrometry: When fringe patterns from multiple sources are well separated (i.e. by multiple coherence lengths) in delay space, then the delay separation between fringe packets contains information on the relative separation of the sources. For fields larger than about 100 millarcseconds the separation between multiple fringe packets in delay space is the observable proxy for sky separation. Operationally various observation and measurement techniques are applied as a function of field size, but the common thread is the delay separation between objects in the field.

\section{Visibility amplitude results}

The first and largest body of scientific results from LB OIR interferometric astrometry follow from visibility amplitude analysis of close binary stars. As mentioned above, such binary star analysis was initially described by Michelson himself (1920), and using his interferometer Anderson (1920) and Merrill (1922) observed a number of binary stars, and published the first orbit determinations for the Capella system. The visibility amplitude effects created by the overlap of multiple fringe packets within a single coherence length are straightforward to compute (see Michelson 1920 and Boden 1999a). Astrometry using this method began with Capella, but has been broadly applied with contributions from most ground-based LB OIR interferometers (e.g. Table 1). Figure 1 illustrates a visual orbit on the nearby binary $\iota$ Pegasi (Boden et al. 1999b) reconstructed from interferometric visibility data from the Palomar Testbed Interferometer (PTI; Colavita et al. 1999). Interferometric visibility measurements serve as proxy for relative astrometry between the components, allowing an estimate of the component separation (and over time the orbit). Modern visibility analysis methods estimate the orbit directly from the visibility data without intermediate separation estimates (e.g. Herbison-Evans 1971 and Hummel 1993), and directly integrate radial-velocity (RV) data when available (e.g. Hummel 1998 and Boden 1999c).

The primary objective in most visibility amplitude/binary analyses is the determination of fundamental parameters (e.g. dynamical mass, radius, luminosity) for the components (a notable exception is work on Atlas by Pan et al. 2004 and Zwahlen et al. 2004 discussed elsewhere in these proceedings). Table 1 (reprinted from Cunha et al. 2007) lists the set of binary systems that have been analyzed using LB OIR interferometric data combined with double-lined RV data. There are a total of 34 systems listed spanning nearly the entire HR-diagram; all but three of these entries (discussed below) use interferometric visibility data as proxy for the component astrometry. Notable in this list are contributions on open cluster stars (e.g. $\theta^{2}$ Tau; Armstrong et al. 2006), pre-main 
Table 1. Interferometrically determined orbits and component masses for double-lined spectroscopic binaries. More details are available in the references listed below the table. The star $\kappa$ Peg is a triple system; in this case the "wide" $(\mathrm{A}-\mathrm{Ba} / \mathrm{Bb})$ and "narrow" $(\mathrm{Ba}-\mathrm{Bb})$ orbits are listed separately. Reprinted with permission from Cunha et al. (2007).

\begin{tabular}{|c|c|c|c|c|c|c|}
\hline System & $\begin{array}{c}\text { Spectral } \\
\text { Types }\end{array}$ & $\begin{array}{c}a^{\prime \prime} \\
{[\mathrm{mas}]}\end{array}$ & $M_{1}\left[M_{\odot}\right]$ & $M_{2}\left[M_{\odot}\right]$ & Instr. & Ref. \\
\hline HD 27483 & $\mathrm{~F} 6 \mathrm{~V}+\mathrm{F} 6 \mathrm{~V}$ & 1.3 & $1.38 \pm 0.13$ & $1.39 \pm 0.13$ & PTI & K04 \\
\hline$\alpha \mathrm{Vir}$ & B1III-IV+B3V: & 1.5 & $10.9 \pm 0.9$ & $6.8 \pm 0.7$ & Narrabri & $\mathrm{H} 71$ \\
\hline$\kappa$ Peg B & $\mathrm{F} 5 \mathrm{IV}+\mathrm{K} 0 \mathrm{~V}:$ & 2.5 & $1.662 \pm 0.064$ & $0.814 \pm 0.046$ & PTI & M06 \\
\hline V773 Tau A & $\mathrm{K} 2+?$ & 2.8 & $1.54 \pm 0.14$ & $1.332 \pm 0.097$ & $\mathrm{KI}$ & B 07 \\
\hline$\theta \mathrm{Aql}$ & $\mathrm{B} 9.5 \mathrm{III}+\mathrm{B} 9.5 \mathrm{III}$ & 3.2 & $3.6 \pm 0.8$ & $2.9 \pm 0.6$ & Mk III & H95 \\
\hline$\beta$ Aur & $\mathrm{A} 2 \mathrm{~V}+\mathrm{A} 2 \mathrm{~V}$ & 3.3 & $2.41 \pm 0.03$ & $2.32 \pm 0.03$ & Mk III & H95 \\
\hline \multirow[t]{2}{*}{12 Boo } & $\mathrm{F} 9 \mathrm{IV}+\mathrm{F} 9 \mathrm{IV}$ & 3.4 & $1.435 \pm 0.023$ & $1.408 \pm 0.020$ & PTI & B 00 \\
\hline & & 3.5 & $1.416 \pm 0.005$ & $1.374 \pm 0.005$ & combined & $\mathrm{B} 05 \mathrm{~b}$ \\
\hline$\sigma \mathrm{Sco}$ & $\mathrm{B} 1 \mathrm{III}+\mathrm{B} 1 \mathrm{~V}$ & 3.6 & $18.4 \pm 5.4$ & $11.9 \pm 3.1$ & SUSI & N07b \\
\hline$\gamma^{2}$ Vel & $\mathrm{O} 7.5 \mathrm{II}+\mathrm{WC} 8$ & 3.6 & $28.5 \pm 1.1$ & $9.0 \pm 0.6$ & SUSI & N07c \\
\hline BY Dra & $\mathrm{K} 4 \mathrm{~V}+\mathrm{K} 7.5 \mathrm{~V}$ & 4.4 & $0.59 \pm 0.14$ & $0.52 \pm 0.13$ & PTI & B01 \\
\hline$o$ Leo & $\mathrm{F} 9+\mathrm{A} 5 \mathrm{~m}$ & 4.5 & $2.12 \pm 0.01$ & $1.87 \pm 0.01$ & combined & H01 \\
\hline HD 9939 & $\mathrm{~K} 1 \mathrm{IV}+\mathrm{K} 0 \mathrm{~V}$ & 4.9 & $1.072 \pm 0.014$ & $0.838 \pm 0.008$ & PTI & B 06 \\
\hline$\sigma \mathrm{Psc}$ & $\mathrm{B} 9.5 \mathrm{~V}+\mathrm{B} 9.5 \mathrm{~V}$ & 5.6 & $2.65 \pm 0.27$ & $2.36 \pm 0.24$ & PTI & K04 \\
\hline $64 \mathrm{Psc}$ & $\mathrm{F} 8 \mathrm{~V}+\mathrm{F} 8 \mathrm{~V}$ & 6.5 & $1.223 \pm 0.021$ & $1.170 \pm 0.018$ & PTI & $\mathrm{B} 99 \mathrm{c}$ \\
\hline 93 Leo & $\mathrm{G} 5 \mathrm{III}+\mathrm{A} 7 \mathrm{~V}$ & 7.5 & $2.25 \pm 0.29$ & $1.97 \pm 0.15$ & Mk III & H95 \\
\hline \multirow[t]{2}{*}{$\zeta^{1} \mathrm{UMa}$} & $\mathrm{A} 2 \mathrm{~V}+\mathrm{A} 2 \mathrm{~V}$ & 9.6 & $2.51 \pm 0.08$ & $2.55 \pm 0.07$ & Mk III & $\mathrm{H} 95$ \\
\hline & & 9.8 & $2.43 \pm 0.07$ & $2.50 \pm 0.07$ & NPOI & H98 \\
\hline$\iota \mathrm{Peg}$ & $\mathrm{F} 5 \mathrm{~V}+\mathrm{G} 8 \mathrm{~V}$ & 10.3 & $1.326 \pm 0.016$ & $0.819 \pm 0.009$ & PTI & B99b \\
\hline$\eta$ And & G8III+G8III & 10.4 & $2.59 \pm 0.30$ & $2.34 \pm 0.22$ & Mk III & H93 \\
\hline$\alpha \mathrm{Equ}$ & $\mathrm{G} 2 \mathrm{III}+\mathrm{A} 5 \mathrm{~V}$ & 12.0 & $2.13 \pm 0.29$ & $1.86 \pm 0.21$ & Mk III & A $92 \mathrm{~b}$ \\
\hline 27 Tau & B8III+? & 13.1 & $4.74 \pm 0.25$ & $3.42 \pm 0.25$ & combined & Z04 \\
\hline HD 195987 & G9V+? & 15.4 & $0.844 \pm 0.018$ & $0.665 \pm 0.008$ & PTI & T02 \\
\hline$\zeta$ Aur & $\mathrm{K} 4 \mathrm{Ib}+\mathrm{B} 5 \mathrm{~V}$ & 16.2 & $5.8 \pm 0.2$ & $4.8 \pm 0.2$ & Mk III & B96 \\
\hline \multirow[t]{2}{*}{$\theta^{2}$ Tau } & $\mathrm{A} 7 \mathrm{III}+\mathrm{A}$ : & 18.6 & $2.1 \quad \pm 0.3$ & $\begin{array}{ll}1.6 & \pm 0.2 \\
1.6 & \pm 0.2\end{array}$ & Mk III & T95 \\
\hline & & 18.8 & $2.15 \pm 0.12$ & $1.87 \pm 0.11$ & combined & A 06 \\
\hline$\lambda$ Vir & $\mathrm{Am}+\mathrm{Am}$ & 19.8 & $1.897 \pm 0.016$ & $1.721 \pm 0.023$ & IOTA & $\mathrm{Z} 07$ \\
\hline HD $98800 \mathrm{~B}$ & $\mathrm{~K} 5 \mathrm{~V}+?$ & 23.3 & $0.699 \pm 0.064$ & $0.582 \pm 0.051$ & KI & B05a \\
\hline$\phi \mathrm{Cyg}$ & K0III+K0III & 23.7 & $2.536 \pm 0.086$ & $2.437 \pm 0.082$ & Mk III & A92a \\
\hline$\alpha$ And & $\mathrm{B} 8 \mathrm{IV}+\mathrm{A}:$ & 25.2 & 5.5: & 2.3 : & Mk III & Р 92, Т 95 \\
\hline$\beta$ Cen & $\mathrm{B} 1 \mathrm{III}+\mathrm{B} 1 \mathrm{III}$ & 25.3 & $11.2 \pm 0.7$ & $9.8 \pm 0.7$ & SUSI & $\mathrm{D} 05, \mathrm{Au} 06$ \\
\hline$\beta$ Ari & A5V+G0V: & 36.1 & $2.34 \pm 0.10$ & $1.34 \pm 0.07$ & Mk III & P90 \\
\hline$\lambda \mathrm{Sco}$ & $\mathrm{B} 1.5 \mathrm{IV}+\mathrm{B} 2 \mathrm{~V}$ & 49.3 & $10.4 \pm 1.3$ & $8.1 \pm 1.0$ & SUSI & T06 \\
\hline 12 Per & $\mathrm{F} 8 \mathrm{~V}+\mathrm{G} 2 \mathrm{~V}$ & 53.2 & $1.382 \pm 0.019$ & $1.240 \pm 0.017$ & CHARA & $\mathrm{Ba} 06$ \\
\hline$\alpha$ Aur & G1III+G8III & 55.7 & $2.56 \pm 0.04$ & $2.69 \pm 0.06$ & Mk III & H94 \\
\hline$\delta \mathrm{Equ}$ & $\mathrm{F} 7 \mathrm{~V}+\mathrm{F} 7 \mathrm{~V}$ & 231.9 & $1.193 \pm 0.012$ & $1.188 \pm 0.012$ & PTI & M05 \\
\hline$\kappa \mathrm{Peg}$ & $\mathrm{F} 5 \mathrm{IV}+\mathrm{F} 5 \mathrm{IV}$ & 235.0 & $1.549 \pm 0.050$ & composite & PTI & M06 \\
\hline
\end{tabular}

References: A92a: Armstrong 1992a; A92b: Armstrong 1992b; A06: Armstrong 2006; Au06: Ausseloos 2006; Ba06: Bagnuolo 2006; B96: Bennett 1996; B99b: Boden 1999b; B99c: Boden 1999c; B00: Boden 2000; B01: Boden \& Lane 2001; B05a: Boden 2005a; B05b: Boden 2005b; B06: Boden 2006; B07: Boden 2007; D05: Davis 2005; H71: Herbison-Evans 1971; H93: Hummel 1993; H94: Hummel 1994a; H95: Hummel 1995; H98: Hummel 1998; H01: Hummel 2001; K04: Konacki \& Lane 2004; M05: Muterspaugh 2005; M06: Muterspaugh 2006; N07a: North 2007a; N07b: North 2007b; P90: Pan 1990; P92: Pan 1992; T95: Tomkin 1995; T02: Torres 2002; T06: Tango 2006; Z04: Zwahlen 2006; Z07: Zhao 2007

sequence stars (e.g. V773 Tau; Boden et al. 2007), low-abundance/Galactic thick-disk stars (e.g. HD 195987; Torres et al. 2002), subgiant (e.g. 12 Boo; Boden et al. 2005b) and giant (e.g. $\alpha$ Equ; Armstrong et al. 1992b) stars, and even triple systems ( $\eta$ Vir; Hummel et al. 2003). Prospects are excellent for continued important contributions in these areas. 

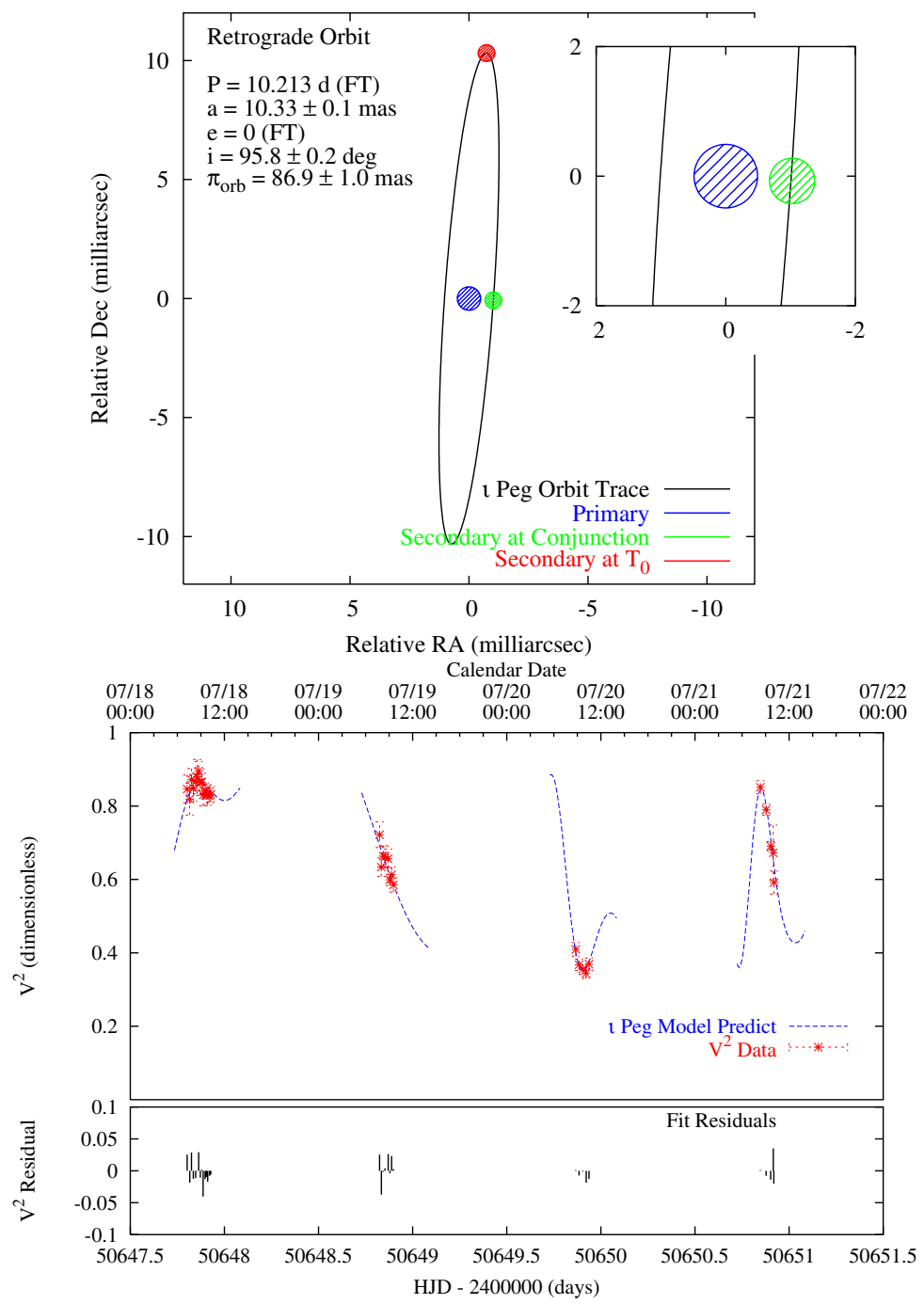

Figure 1. Visual Orbit of $\iota$ Pegasi and Supporting Visibility Data (from Boden (1999b)). Top: the relative visual orbit of $\iota$ Pegasi, with the primary rendered at the center, and the secondary position shown at both maximum elongation and conjunction. The $\iota$ Peg orbit is in fact circular but seen at a near-eclipsing orientation. Bottom: four consecutive nights of PTI calibrated visibility $\left(\mathrm{V}^{2}\right)$ data on $\iota$ Peg. Variations in the measured visibility can be used as proxy for the relative separation between the components.

\section{Separated fringe-packet astrometry}

Fringe amplitude astrometry techniques necessarily require the multiple sources to be within a single interferometric coherence length, typically on the order of tens to $\approx 100$ mas projected on the sky. When astrometric fields increase beyond this 100 mas, multiple fringe packets are well-separated in interferometric delay space, and the delay difference itself becomes the observable proxy for relative astrometry. Above we have described such astrometry as separated fringe packet astrometry.

As a practical matter, separated fringe packet astrometry further subdivides into three different operating regimes that we will discuss in turn. 


\subsection{Very narrow angle astrometry}

When the astrometric field is still within a interferometer sub-aperture (single telescope) diffraction pattern (e.g. $\approx 1$ arcsec), then the multiple fringe packets can be observed simply by scanning the delay compensation device (typically a "delay line"; Colavita et al. 1999). By measuring the delay scan distance separating the multiple fringe packets (typically by laser metrology) one obtains an astrometrically-useful delay difference estimate. The first results of this kind are presented in Colavita (1994), and this method is described in detail in Lane \& Muterspaugh 2004 (including performance improvements from synthetic phase stability allowed by the unique PTI dual-beam/fringe tracker design). When phase referencing is available typical relative astrometric precisions are on the order of $15 \mu$ arcseconds over a 0.5 arcsecond field (a fractional precision of 3 parts in $10^{5}$ ). Astrophysical results from this method are included in the Table 1 compilation: $\delta$ Equ (Muterspaugh et al. 2005), $\kappa$ Peg (Muterspaugh et al. 2006), and 12 Per (Bagnuolo et al 2006). Further, such astrometric precision enables planet-search programs, and the PTI PHASES astrometric planet search program is discussed in Muterspaugh et al. (2006) and Muterspaugh et al. (2007)

\subsection{Dual-beam narrow-angle astrometry}

While astrometric precisions at the arcsecond-scale are remarkable, the small working angles practically dictate that the methods are limited to arcsecond-scale binaries. A method to increase this field to expand sky coverage was presented by Shao \& Colavita (1992a). In this narrow-angle astrometric scenario the fringes on multiple stars are tracked simultaneously to take advantage of significant correlation in the atmospheric phase noise over an isoplanatic angle $(\approx 30-40$ arcsec in the near-IR; Quirrenbach 1999). The simultaneous fringe tracking requirement necessitates multiple beam combiners in a single facility, and guided the dual-fringe tracker design of PTI (Colavita et al. 1999). Hence this technique has been termed dual-beam narrow-angle astrometry.

This dual-beam astrometric technique has been demonstrated at PTI. Figure 2 shows the results of a three-months demonstration experiment performed in 1999 using the well-studied, nearby visual binary 61 Cygni. 61 Cyg is a nearby K-dwarf binary with an estimated 678-year orbital period and apparent separation at present epoch of roughly 31 arcseconds (Gorshanov et al. 2006). Using the PTI N-S baseline (so the principle measurement axis is in declination) PTI collected narrow-angle astrometric data on 61 Cyg over a three-month period in 1999. These astrometric measurements are made by tracking fringes on the two stars with two independent fringe trackers, and relating the relative delay between the two fringe trackers using a laser metrology system (details are given in Colavita et al. 1999). Projecting the derived 2-d separations into a space of declination vs time, the apparent night-to-night separation increase is clearly seen. Fitting a linear motion model to these separations, the ensemble RMS declination residual is $170 \mu$ arcseconds, with a particularly stable, contiguous 7 -night run showing a 100 $\mu$ arcseconds scatter around the same ensemble motion model. This scatter represents a fractional precision of 3 parts in $10^{6}$. Application of this method to ground-based astrometric planet searches is planned for the VLTI PRIMA instrument, discussed in detail elsewhere in these proceedings (e.g. see contributions by Richichi and Launhardt).

\subsection{Wide-angle astrometry}

The narrow-angle method of Shao \& Colavita (1992a) leverages the significant correlation in atmospheric turbulence over an isoplanatic angle (30-50 arcseconds at a typical site) to make 10-100 $\mu$ arcseconds-class relative astrometric measurements. For interferometric measurements over a wider field this correlation is not applicable. Such wide-angle 


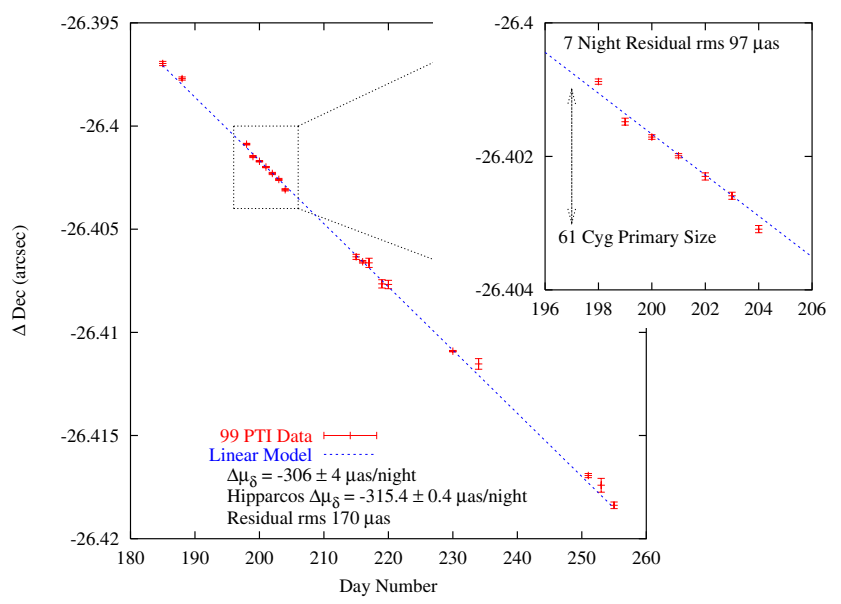

Figure 2. Principle Projection for 1999 PTI Dual-Beam Narrow-Angle Astrometry Demonstration. Roughly three months of relative astrometry data on the 30 -arcsecond visual binary 61 Cygni are shown projected onto the principle (declination) measurement axis (all astrometric data was taken with the PTI N-S baseline) vs time. The apparent night-to-night relative motion due to the estimated 678-year orbit period is clear in these data. The apparent relative declination motion of $300 \mu \mathrm{arcsecond} /$ day is very close to that measured by the Hipparcos satellite (the difference between the two estimates is consistent with acceleration from the binary orbit). The RMS scatter of these data around the best-fit linear motion model is $170 \mu$ arcsecond; data from a particularly stable 7 -night run (inset) shows an RMS scatter of approximately $100 \mu$ arcsecond

measurements have been demonstrated by the Mark III interferometer (Shao et al. 1988) and Navy Prototype Optical Interferometer (NPOI; Armstrong et al. 1998).

Mark III wide-angle astrometry work is described in Mozurkewich et al. (1988), Shao et al. (1990), and Hummel et al. (1994b). This work focused on making serial delay measurements on a set of roughly 20 FK5 stars over a number of years, and using the data to compute differential corrections to the mean-epoch positions from the FK5 catalog. Shao et al. (1990) reports single-season RMS residuals of individual measurements on the order of 10 milliarcseconds, with Hummel et al. (1994b) reporting four-year ensemble RMS precisions on the order of 20 milliarcseconds. Over a wide-angle field on the order of 1 radian these measurements represent a fractional single-night precision of 1 part in $10^{7}$.

Based on the success of Mark III wide-angle program, the NPOI was constructed to allow wide-angle astrometry over an extended period, with the objective of extending the proper-motion time baseline for Hipparcos stars. Benson et al. (2004) and Johnston et al. (2006) report on NPOI astrometry program status. At present NPOI astrometry is demonstrating 10-millarcsecond-class astrometric measurement precision in single-night runs, and the NPOI team anticipate multi-night ensemble astrometric solutions to be completed in the next year. NPOI wide-angle astrometry holds the promise to significantly improve the precision of Hipparcos proper motion and parallax estimates over the next few years, particularly in advance of Gaia (discussed elsewhere in these proceedings by Lindegren and Mignard).

\section{Summary}

We have summarized astrometric contributions by LB OIR interferometers. To date the largest body of results have focused on analysis of binary systems with an eye toward 
stellar fundamental parameter determinations, and interferometers will continue making important contributions in these areas (e.g. see Cunha et al. 2007 for a discussion). Further, the VLTI PRIMA facility is planning a broad astrometric exoplanet search program (see other contributions by Richichi and Launhardt in these proceedings); prospects are excellent for this (and other) astrometric survey(s) to contribute to understanding of the architecture and diversity of planetary systems.

\section{Acknowledgments}

We are happy the thank the organizers of IAUS 248, who graciously opened their city, country, and institution to all of us during the symposium. Further, we thank H. McAlister, D. Hutter, J. Benson, and K. Johnston for their advice and contributions in the preparation of this manuscript and presentation.

\section{References}

Anderson, J. 1920, ApJ, 51, 263.

Armstrong, J. et al. 1992a, AJ, 104, 2217.

Armstrong, J. et al. 1992b, AJ, 104, 241.

Armstrong, J. et al. 1998, ApJ, 496, 550.

Armstrong, J. et al. 2006, AJ, 131, 2643.

Ausseloos, M. et al. 2006, A\&A, 455, 259.

Bagnuolo, W. et al. 2006, ApJ, 131, 2695.

Bennett, P. et al. 1996, ApJ, 471, 454.

Benson, J. et al. 2004, SPIE, 5491, 464.

Boden, A. 1999a, in Proc. 1999 Michelson Summer School, Lawson, P. editor (http://sim.jpl. nasa.gov/michelson/iss1999/index.html).

Boden, A. et al. 1999b, ApJ, 515, 356.

Boden, A. et al. 1999c. ApJ, 527, 360 .

Boden, A. et al. 2000, ApJ, 536, 880.

Boden, A. \& Lane, B. 2001 ApJ, 547, 1071.

Boden, A. et al. 2005a, ApJ, 635, 442.

Boden A., Torres G. \& Hummel C. 2005b, ApJ, 627, 464.

Boden, A., Torres, G. \& Latham, D. 2006. ApJ, 644, 1193.

Boden, A. et al. 2007, ApJ, 670, 1214 (astro-ph/0706.2376).

Colavita, M. 1994, A\&SA, 283, 1027.

Colavita, M. et al. 1999, ApJ, 510, 505.

Cunha et al. 2007, Ast $\mathcal{E}$ Astrop Rev in press (astro-ph/0709.4613).

Davis, J. et al. 2005, MNRAS, 356, 1362.

Gorshanov, D., Shakht, N., \& Kisselev, A. 2006, Ap, 49, 386.

Herbison-Evans, D. et al. 1971, MNRAS, 151, 161.

Hummel, C. et al. 1993 AJ, 106, 2486.

Hummel, C. et al. 1994a, AJ, 107, 1859.

Hummel, C. et al. 1994b, AJ, 108, 326.

Hummel, C. et al. 1995, AJ, 110, 376.

Hummel, C. et al. 1998, AJ, 116, 2536.

Hummel, C. et al. 2001 AJ, 121, 1623.

Hummel, C. et al. 2003 AJ, 125, 2630.

Johnston, J. et al. 2006, SPIE, 6268, 6.

Konacki, M. \& Lane, B. 2004, ApJ, 610, 443.

Lane, B. \& Muterspaugh, M. 2004, ApJ, 601, 1129.

Merrill, P. 1922, ApJ, 56, 40.

Michelson, A. 1920, ApJ, 51, 257. 
Mozurkewich, D. et al. 1988, AJ, 95, 1269.

Muterspaugh, M. et al. 2005, AJ, 130, 2866.

Muterspaugh, M. et al. 2006a, ApJ, 636, 1020.

Muterspaugh, M. et al. 2006b, ApJ, 653, 1469.

Muterspaugh et al. 2007, in "Planets in Binary Systems", N. Haghighipour Editor (Springer) (astro-ph/0705.3072).

North, J. et al. 2007a. MNRAS, 380, 1276.

North, J. et al. 2007b, MNRAS, 377, 415.

Pan, X. et al. 1990, ApJ, 356, 641.

Pan, X. et al. 1992, ApJ, 384, 624.

Pan, X., Shao, M., \& Kulkarni, S. 2004, Nature, 427, 326.

Perley, R., Schwab, F., \& Bridle, A. 1989, Synthesis Imaging in Radio Astronomy, ASP Conf. Series 6 (Provo UT: Brigham Young Univ. Press).

Quirrenbach, A. 1999, in Proc. 1999 Michelson Summer School, Lawson, P. editor (http://sim. jpl.nasa.gov/michelson/iss1999/index.html).

Quirrenbach, A. 2001, ARA\&A, 39, 353.

Shao, M. et al. 1988, ApJ, 327, 905.

Shao, M. et al. 1990, AJ, 100, 1701.

Shao, M. \& Colavita, M. 1992a, A\&SA, 262, 353.

Shao, M. \& Colavita, M. 1992b, ARA\& $\&$, 30, 457.

Tango, W. et al. 2006, MNRAS, 370, 884.

Thompson, R, Moran, J., \& Swenson, G. 2001 Interferometry and Synthesis on Radio Astronomy 2nd Edition, (New York: John Wiley \& Sons).

Tomkin, J., Pan, X., \& McCarthy, J. 1995, ApJ, 109, 780.

Torres, G. et al. 2002, AJ, 124, 1716.

Zwahlen, N. et al. 2004, A\&SA, 425, L45.

Zhao, M. et al. 2007, ApJ, 659, 626. 